\title{
THE TUBERCULOUS KIDNEY. DIAGNOSIS AND PROGNOSIS
}

\author{
By CHARLES WELLS, F.R.C.S. \\ (Dept. of Surgery, University of Liverpool; Royal Liverpool United (Royal Southern) Hospital)
}

\section{Introduction}

The background of this paper is a series of rather less than a hundred personal cases of renal tuberculosis. The full series will be published elsewhere with, in each case, brief notes of clinical history, findings, treatment and progress. Here are presented the conclusions reached from a study of these records. Illustrative cases are quoted out of the main text which may, therefore, be read through without interruption.

\section{Presenting Features}

Increased frequency of micturition, epididymitis, tuberculous "bacilluria," haematuria, renal colic and an accidental X-ray finding. These, in this order, are the presenting features in renal tuberculosis. By far the most important is increased frequency of micturition, which, when persistent and unremitting over a long period, is almost diagnostic, especially if associated with pain and urgency when the bladder is full. In such cases the classical finding of pus cells in a "sterile," acid urine takes the diagnosis a step further. Parenthetically, we may note that these symptoms with a clear specimen are strongly suggestive of Hunner's ulceration an important, not uncommon, frequently missed, and most distressing disease to which I shall refer again.

Increased frequency is attributable to one or other of four degrees of change in the bladder. These are (I) superficial cystitis in which individual tubercles may be seen in various stages of integration and disintegration; (2) ulcerative or proliferative cystitis; (3) fibrosis of the bladder wall with diminished capacity; (4) fibrosis of the bladder neck with residual urine. In the first two of these four degrees one can look forward to the recovery of normal bladder function and the complete disappearance of tubercle bacilli from the urine. Once the bladder has suffered intrinsic change, some degree of permanently diminished capacity is an inevitable sequel, and deep ulceration and extension of the disease to the prostate and vesicles will determine the continued appearance of pus cells and tubercle bacilli in the specimen long after nephrectomy (see case $I_{7}$ ). This finding, therefore, need not be regarded as an indication of disease in the remaining kidney. In general, the capacity for recovery far exceeds one's expectation (see case I), and continued troublesome frequency is exceptional in any but the most neglected cases in which the thimble bladder has been approached before nephrectomy is undertaken.

The amelioration of frequency and vesical pain is the goal at which treatment is aimed, and the degree by which frequency is diminished serves as an index of success or failure, especially in the patient's view! All other criteria must be measured by one or other of the means of investigation at our disposal-X-ray, cystoscopy, urinalysis, blood chemistry, etc.

Fpididymitis is a danger signal of renal tuberculosis which has in the past been too often neglected. In my experience it is easily the second most frequent presenting feature, and every patient with tuberculous epididymitis should be submitted to renal investigation (see cases I, 2, 3, and 4). In well over 5ó per cent of cases a surgical lesion of the kidney can be found. Personally I hold the view that epididymitis is, for all practical purposes, due to spread of infection from the urinary stream along the lumen of the vas. (1)

Tuberculous epididymitis, therefore, calls not only for local treatment, but also for renal investigation. If the latter proves negative it should be repeated in six months and again intwelve months, and observation should be kept upon the patient for an indefinite period. Aetiologically, epididymitis is closely related to tuberculous "bacilluria," together with which it must be considered.

Tuberculous "bacilluria" has for long been regarded with lively interest by all observers who have had much to do with tuberculous patients. The odd behaviour of individual patients (see case 5) leaves the door open for doubt that the last word has yet been said and much confusion still remains, but there is little real excuse for it. The intermittent appearance of tubercle bacilli in the urine of patients known to be suffering from tuberculosis other than in the genitourinary tract is a well-recognised phenomenon. Such showers of bacilli are always accompanied by pus cells. When the patients concerned are investigated urologically, only a percentage are found to be suffering from demonstrable surgical lesions. Mediar(2) and Band(3) 

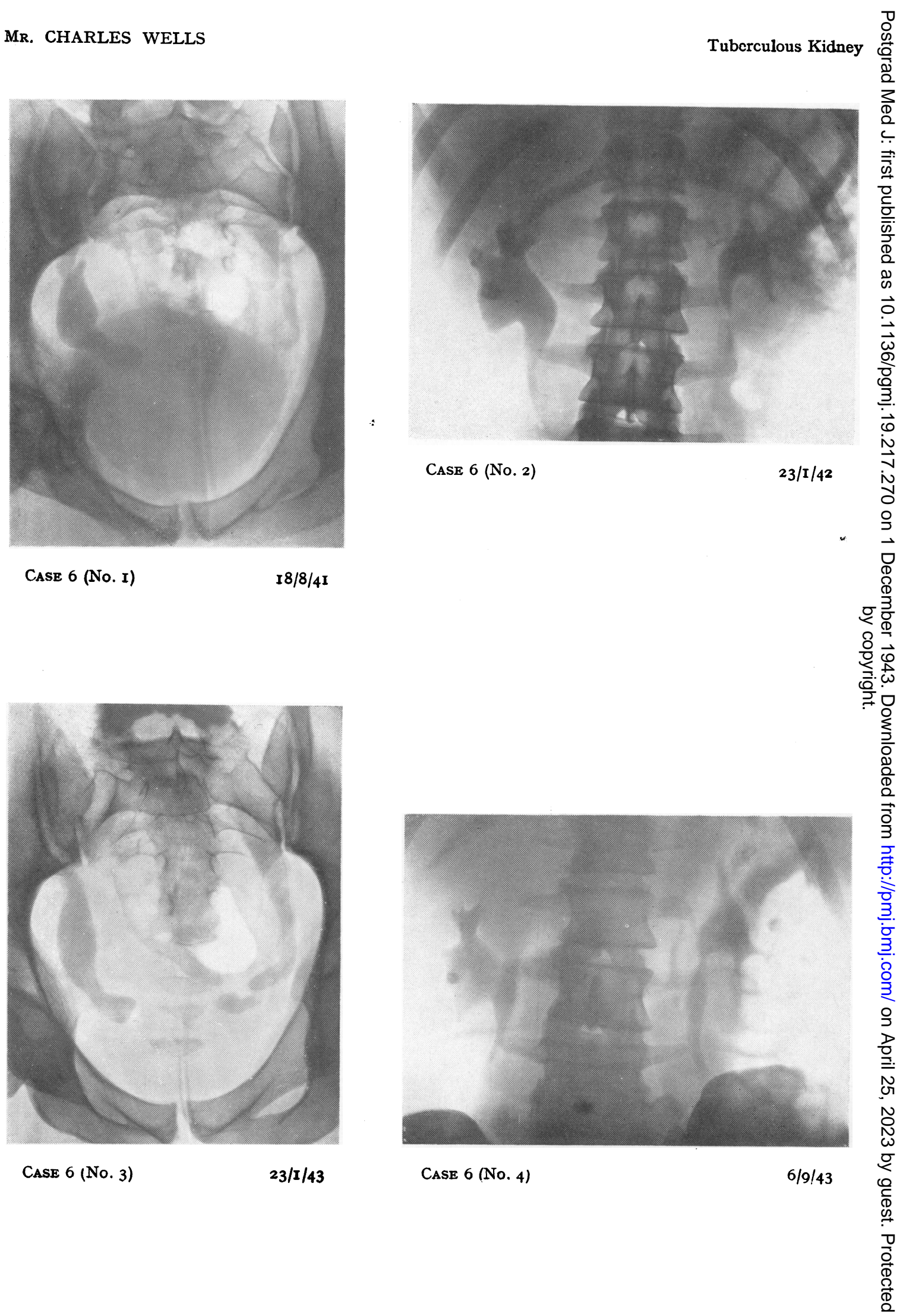


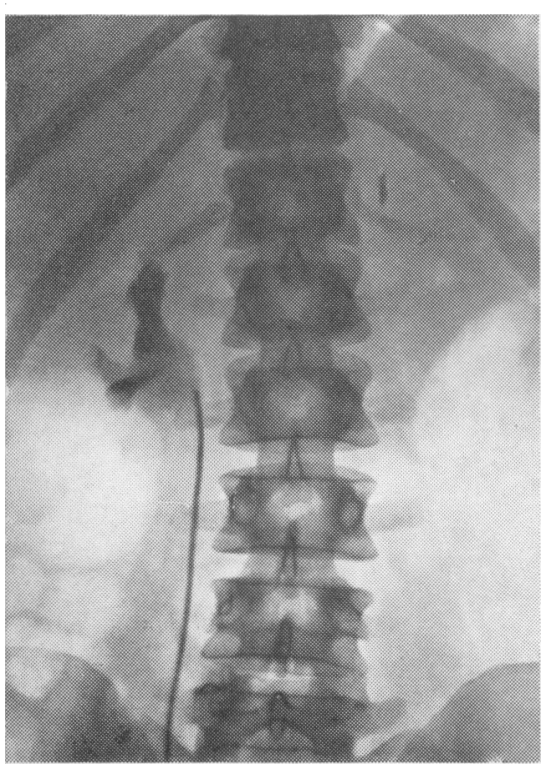

Case 7 (No. I)
$13 / 8 / 42$

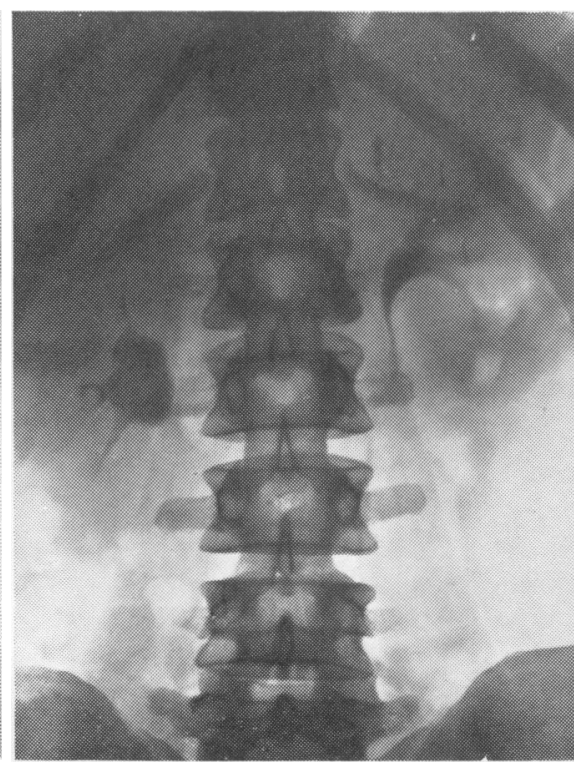

CASE 7 (No. 2)
$18 / 8 / 42$
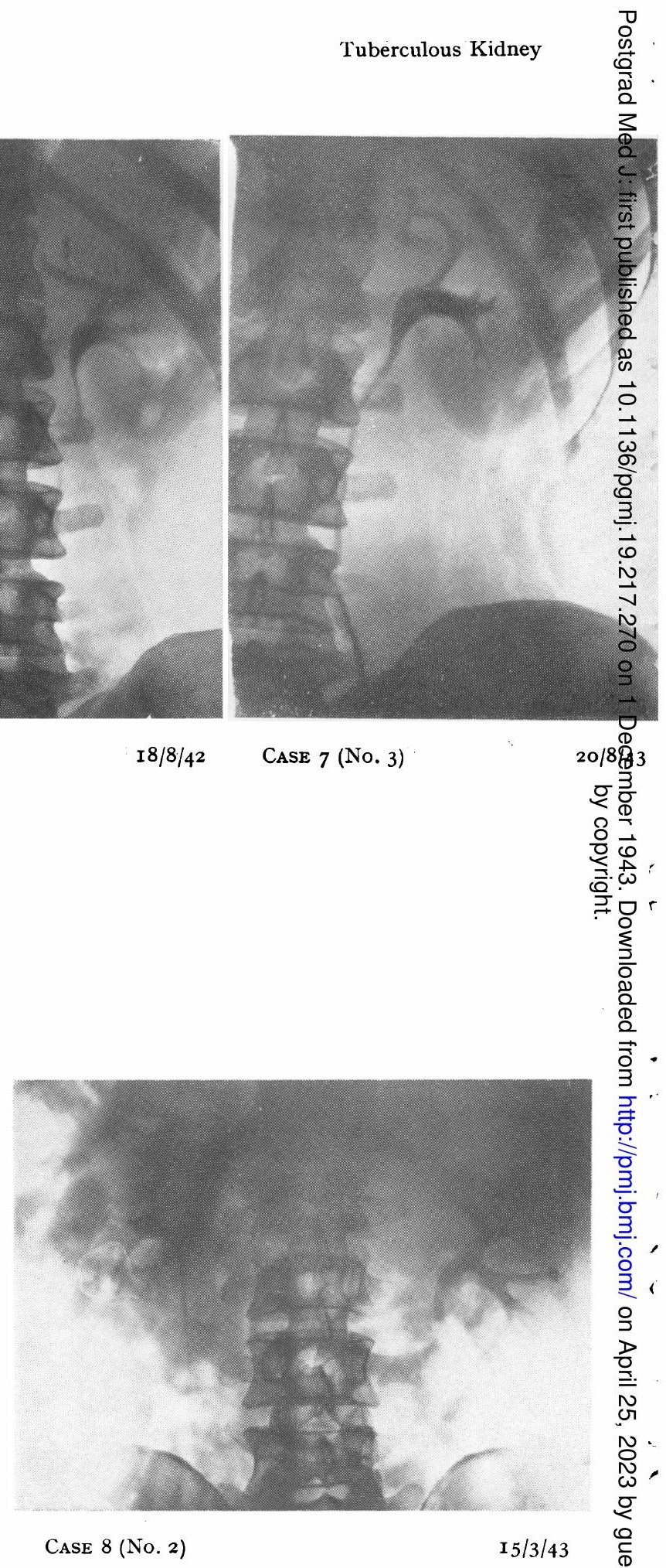

20/8 3

웅

음 름 흔. 망

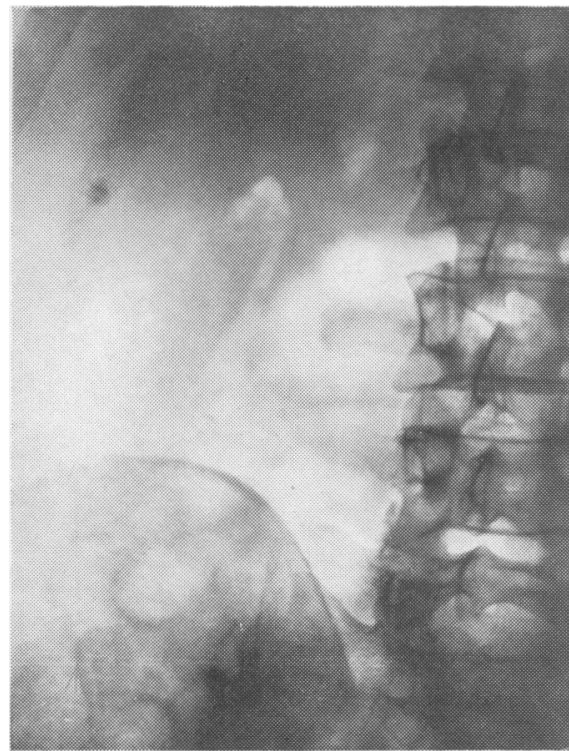

CASE 8 (No. I)

I $5 / 3 / 43$

CASE 8 (No. 2) 
and others,(4) however, have followed to post mortem a number of these patients in whom no gross surgical renal lesion could be demonstrated and have found in the kidneys evidence of healed microscopic foci. The recognition of this work is an essential step in the logical interpretation of the commonplaces of tuberculosis of the genito-urinary tract. We are, for example, at once afforded an explanation of the sources of infection in tuberculous epididymitis not associated with a surgical lession in the kidney, without having to postulate any other route than the lumen of the vas.

The appearance of tubercle bacilli in the urine without related symptoms is brought to the notice of the urologist in two groups of cases; first, those known to be tuberculous, characteristically the sanatorium group; and secondly, those in whom the finding is wholly unexpected. The latter naturally come from relatively unskilled observers, and one is often tempted to regard with suspicion the reported evidence. Perhaps the most important source of error is the coldwater tap saprophyte described by Brem (5) (IgO9) and Reitzke (6) (I9IO). Morphologically and by staining this is indistinguishable from the tubercle bacillus. The mistake is not likely to be made by an experienced bacteriologist. In any case of doubt the finding can'be checked by culture or by guinea-pig inoculation if the original specimen is still available.

Haematuria and renal or ureteric pain may occur at any stage of the disease, and their early occurrence clearly depends upon the accidental early erosion of a vessel of some size, the pain in most cases being attributable to the passage of clots.

\section{Investigation}

Bearing in mind the necessity for regarding the patient as a whole, searching for a primary lesion èlsewhere and considering the vitality and resistance of the total organism, the further detailed investigation may be considered under three heads-X-ray, cystoscopy, and urinalysis.

In my opinion, the following is the optimal sequence. (I) Examination of a clean specimen confirmed, in the woman's case, by a catheter specimen if necessary; (2) straight X-ray; (3) intravenous pyelography; (4) cystoscopy, elaborated as necessary.

\section{Urinalysis.}

A persistent trace of albumen in a clinically doubtful case should incline one to further investigation. Repeated, centrifuged specimens may be examined for tubercle bacilli, and either guinea-pig inoculation tests carried out or cult res put up. A limited experience of culture on Loewenstein's medium has left me with a favourable impression, but in a test of such vital importance to the patient I still feel more content with the well-tried guinea-pig test. A careful pathologist will often avoid the sacrifice of both animal and time by reporting the presence of typical acid- and alcohol-fast bacilli in the specimen.

Mixed infections may obscure and delay the diagnosis (see cases 3 and 6). In all cases of infective disease of the urinary tract in which the response to treatment is disappointing, it is well to review the data afresh with the possibility of tuberculosis in mind.

\section{X-ray.}

A straight-through X-ray may appear normal in which case it is of purely negative value. An enlarged, caseated kidney may show a big shadow without calcification or, more ccmmonly, scattered calcification within the renal $s$ hadow may indicate tuberculous disease. In doubtful cases, X-rays made upon inspiration and expiration confirm the intimate and constant relationship between the calcification and the renal outline. In other cases suspected of tuberculosis, stone shadows may appear in the radiograms. These cases must be the subject of special warning, for it is quite possible to find stone in association with tuberculosis, both in sporadic cases (see cases 7 and 8) and in sanatorium cases (see case 9), in which the calculi are most probably of decubitus origin. Grave errors both of diagnosis and procedure may result from their hasty assessment.

Intravenous pyelography is of the greatest possible value in forming a preliminary or even a final opinion. X-rays of first-class quality in a suitable subject may afford all the information necessary for a final diagnosis and a decision to operate. This decision depends, of course, upon the recognition of a destructive lesion on one side and a healthy kidney on the other.

When the intravenous pyelograms are insufficiently informative, retrograde pyelograms 
should be made in order to establish the doubtful points. In this way one may be called upon to demonstrate either the normal anatomy of the supposedly healthy or the morbid anatomy of the supposedly diseased side or both. I have never seen the slightest harm result from retrograde pyelography in these cases, and the demonstration of the anatomy of the upper tract is the most important single step in their detailed diagnosis.

An early destructive tuberculous lesion of the kidney may be recognised by erosion of one or more calyces (see cases 7,8 ). In later stages of the disease cavities appear in the substance of the kidney, and finally there may be a diffuse hydronephrotic change with irregularity of the dilated calyces, or the whole organ may become functionless, with or without calcification. Hydronephrosis with hydro-ureter calls for nephro-ureterectomy.

The completely functionless kidney may constitute a closed lesion, the change being sometimes described as auto-nephrectomy The term is, of course, misleading, since the dead kidney remains a potential source of infection (see case 4). It is cases such as this which are discovered accidentally by X-ray, and there may be no associated symptoms or urinary findings. An interesting possibility is the spontaneous exclusion of just a part of the pelvis and kidney from the remainder of the organ. This causes a filling defect radiologically, the appearances similating those of growth (see case I0). In these cases one may sometimes trace a remote history of renal tuberculosis followed by a prolonged remission of symptoms, and a recent recrudescence seemingly attributable to the breaking down of the barrier between the excluded diseased and the remaining healthy parts of the kidney.

The possible changes on the apparently healthy side are of not less importance. The most important variation is due to constriction of the lower end of the ureter in the thickened and inflamed wall of the bladder. This may cause a notable degree of hydro-ureter and even of hydronephrosis. Cases of this character are often mistakenly regarded as examples of bilateral disease. It is important, therefore, to distinguish between true back-pressure hydronephrosis on the one hand and tuberculous hydronephrosis with erosion on the other (see cases II and I2).

In this connection it is essential to consider the problem of the spread of renal tuberculosis. All our clinical observation is opposed to the conception of spread at a late stage of the disease from one kidney to the other. Our evidence is that tuberculous infection does not extend up the ureter from the bladder. It follows, therefore, that bilateral disease is either present from the beginning or due to a purely co-incidental blood-borne infection of the previously healthy kidney. Disregarding the latter possibility, it follows that a case of long standing is most probably unilateral. It is in just such a case that the bladder is likely to be seriously contracted, and the healthier ureter constricted and dilated. Established bilateral disease is the exception and is found in my series in not more than 5 per cent of the cases in which positive diagnosis of tuberculosis of the kidney was reached.

\section{Cystoscopy.}

In cases in which the intravenous pyelograms show advanced disease on one side and a completely normal kidney on the other, cystoscopy is not a necessary step in diagnosis or assessment to operation. In such cases, however, it is reassuring to see a normal orifice on the healthy side and by means of segregation we may obtain much interesting information. I cannot however too strongly stress my conviction that in the presence of a completely normal pyelogram a positive specimen from the supposedly healthy side should be disregarded and the frankly diseased organ $r \in$ moved. I have watched for years a small group of patients in whom a positive specimen was separated from the sound side in such circumstances (see case I3). Further positive specimens have been recovered repeatedly, but no otherwise demonstrable lesion has developed. On the contrary, in cases in which I have removed a grossly diseased kidney in spite of a slightly doubtful pyelogram on the better side, I have more than once seen the disease progress to a fatal termination.

In tuberculosis, as in most other forms. of cystitis, the changes are more advanced in the floor of the bladder than in its roof, and one is liable to overlook changes in the vault. Hunner's ulceration in particular may be missed in this way, because the trigone and orifices are quite normal. It is only when the eye is turned upwards that one sees the characteristic appearance -rather like an angry scratch, straggling downwards from the region of the air-bubble and shedding large numbers of minute droplets of blood in a little shower when the bladder is overdistended. This change so closely simulates tuberculosis in its symptomatology and cysto- 
scopic appearances-although not in its distribution-that it must be noted in differential diagnosis.

I have only twice (see case I4) seen a completely normal bladder before operation in a case of proved renal tuberculosis. Positive findings are the rule. The earliest change may be a few tubercles grouped round the orifice on the affected side (see case I5). It is usual for cystitis to develop from this area, and the subsequent appearances are widely varied. Irritability and later contraction of the bladder are usual. Gradually the orifice on the affected side loses its supple resilience and becomes indurated and ulcerated. This is the golf-hole ureter. Simultaneously, as the ureter shortens, the orifice gets drawn to a higher and ever higher level in the bladder.

In the worst cases of cystitis very little of this may be visible, and here it is well to have resort at once to intravenous pyelography and urinalysis if these things have not already been done. If there is mixed infection, or if for any reason the truly tuberculous nature of the condition is in doubt, the effect of treatment by antiseptics and by lavage should be essayed, but in my experience it is unwise to leave an in-dwelling catheter in tuberculous cases except in conjunction with tidal drainage (Munro (7) (I936), Wells (8) (I942)), and even then it may have to be removed if poorly tolerated. Any rapid improvement in the cystitis suggests that the cause is not wholly or, more likely, not at all tuberculous.

Hypertrophic reactions to tuberculosis are not unusual, and in such cases the diagnosis from growth may be difficult. The presence of recognisable tubercles somewhere in the picture must then be sought and bimanual palpation under anaesthesia for any mass is of first-class importance in forming an opinion about a possible growth.

In the final stages of tuberculous cystitis the "thimble-bladder" may defeat all attempts at cystoscopy, and other methods of diagnosis will have to be relied upon entirely.

Dye excretion is helpful and informative, but not diagnostic, since a grossly diseased kidney may still excrete indigo-carmine strongly if the greater part of its substance remains intact.

Segregation is generally employed to determine the presence or absence of infection rather than for the estimation of differential function. In general, it is more important to obtain a specimen from the supposedly healthy side. Retrograde pyelography may similarly be needed for confirmation of disease or freedom from disease, and, more often than not, it is the healthier side which needs to be displayed.

\section{Summary and Assessment to Operation}

The criterion for operation is an anatomically intact kidney on one side and a demonstrably diseased one on the other. Intravenous, with or without retrograde, pyelography provides the most valuable single piece of evidence. Supporting evidence is afforded by urinalysis, cystoscopy and segregation.

In long-standing cases, dilatation of the ureter on the good side is likely to be due to fibrosis and not to be indicative of contralateral disease. In early cases, any change on the good side must be regarded as suspicious of bilateral disease, and operation may be delayed for re-examination, comparison and contrast.

Once a decision has been reached on these points, operation is indicated without delay except for the presence of epididymitis, vesiculitis and prostatitis, complications which may lead to abscess or fistula and on account of which the tempo of treatment may be slowed down. Otherwise the time for prolonged sanatorium treatment is after rather than before the operation.

Occasionally, back pressure on the good side may call for temporary nephrostomy. Subsequently the lower end must be slit up or the ureter transplanted into the colon (see case II).

\section{Clinical and operative findings}

The days of transcoelomic exploration as a deliberate measure are over, but it may still be helpful when confronted with a previously undiagnosed tuberculous kidney to pass one's hand across the abdominal cavity. In general, palpation of the kidney is less informative than palpation of the ureter, which becomes noticeably thickened in most cases early in the disease. I do not recommend puncture of the ureter exposed by open operation for a specimen in cases which cannot be catheterised. The necessity and justification for this dangerous measure went out with the introduction of Uroselectan. 
The exact procedure to be followed at operation depends upon the precise findings. If the ureter is dilated and obstructed at its lower end, it must be removed in toto by nephroureterectomy carried out through two incisions. Otherwise a percentage of wound fistulae will follow. If the ureter is not wholly removed it should be fixed in the skin if at all thickened. In the rare cases in which the ureter is thickened and so densely adherent that it cannot safely be removed or brought up to the skin, a tube or catheter should be fixed in its lumen and brought out at the lower angle of the wound (see case I6). This, if retained for three or four weeks will determine the formation of a controllable fistula which may subsequently be expected to heal. I am firmly of the opinion that it is the treatment of the ureter which influences the incidence of post-operative wound infection and fistula formation. Even the most prolonged treatment in a sanatorium will not offset the harmful effect of a widely dilated ureter discharging its infected content into the kidney bed. In the whole of my series, only two patients developed an infected wound, and of these only one was really troublesome. This was a stout woman in poor health in whom I hesitated to remove the ureter with the kidney, although it was clearly desirable to do so, and at that time I had not realised the possibility of direct drainage of the ureteric stump.

\section{Prognosis}

It is clear from what has been said that the outlook in any individual case depends upon such a large number of factors that the statement of prognosis is difficult. Statistics can be applied only after correction for a number of factors, and the making of a forecast is a matter rather of art and experience than of the application of a formula. Certain things can, however, be said.

I. The recovery from operation is uniformly good. The operative mortality and the mortality within the first few post-operative weeks is, or should be, almost nil.

2. In any reasonably straightforward case the outlook is excellent. Bladder function returns to normal or about normal within twelve months as a rule. Apart from other foci of disease the patients are able to return to full work.

3. If the appearance of disease in the kidney is but an incident in the tuberculous history of the patient, the persistence or reappearance of the disease elsewhere may well prove fatal or crippling.

4. The risk of tuberculosis developing in the remaining kidney is small unless it was presentco and unrecognised at the time of investigation. In this category, the long-standing case is the ? better risk because the long history implies the presence of one good kidney.

5. Anuria from intercurrent disease in the remaining kidney is a rare though important occurrence. Stenosis, stone or pyogenic infection may arise (see case 16 ).

6. The prognosis as regards frequency depends upon the degree of bladder involvement at the time of operation.

(a) In superficial cystitis it is excellent. Improvement may continue for as long as two years, at the end of which time, or a shorter time, normal or almost normal micturition is re-established. In cases in which increased frequency persists in spite of a good bladder capacity, dilatation of the bladder neck, resection of the bladder neck, and/or zinc ionisation of the bladder (also useful in Hunner's ulcer) may give relief (see case I8).

(b) Persistent cystitis due to a mixed or intercurrent infection calls for appropriate investigation and treatment. A period of tidal drainage may help enormously. Vesical calculi may form and must be crushed and evacuated.

(c) The small fibrosed bladder entails a permanent legacy of increased frequency. Presacral neurectomy is clearly inadequate. Transplantation of the ureter to the colon (see case I9) gives enormous relief for which patients are most grateful, but the risk is high, and the danger very real of uraemia supervening in about a year or two. Cutaneous ureterostomy is said to be safer, but I have had trouble from sloughing of the ureter and cannot from my own experience advise it.

CASE r.

\section{Case Reports}

Originally seen on account of epididymitis, this patient admitted to frequency, and was found to have a deeply ulcerated bladder, secondary to tuberculosis of the right kidney. A nephro-ureterectomy and epididymectomy were done, and and a very guarded prognosis was given. The recovery was rapid, and after a while the patient married and has since enjoyed excellent health, and now has a large family. 


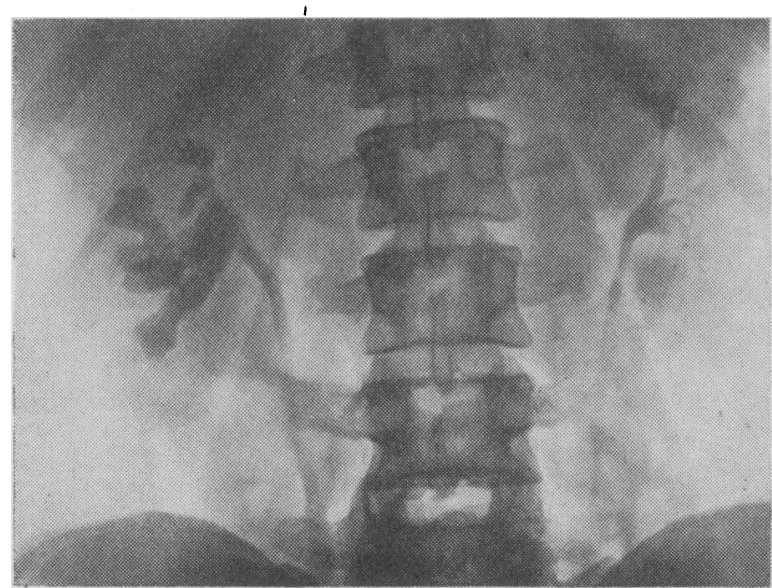

Case ro

I $8 / 1$ I $/ 43$
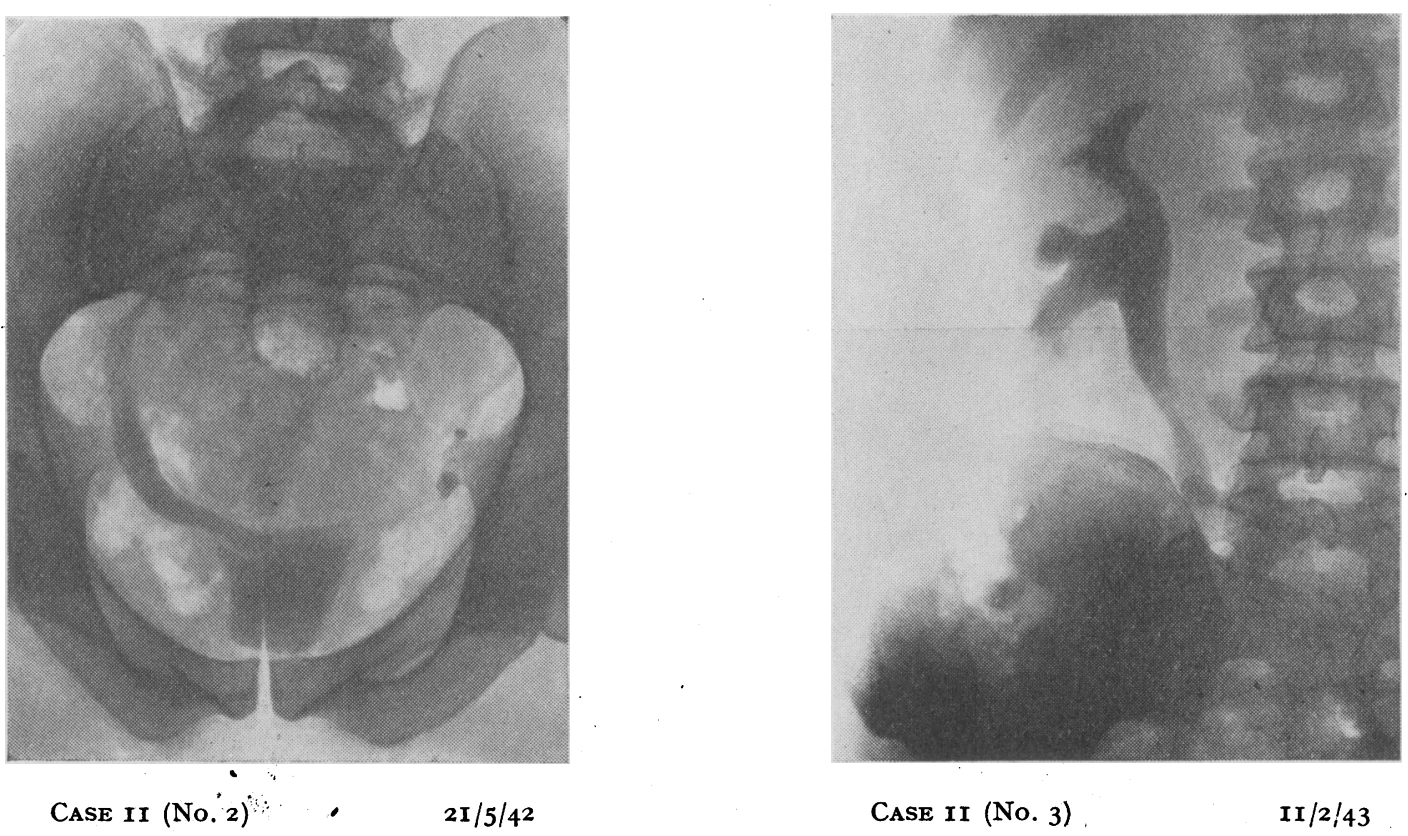

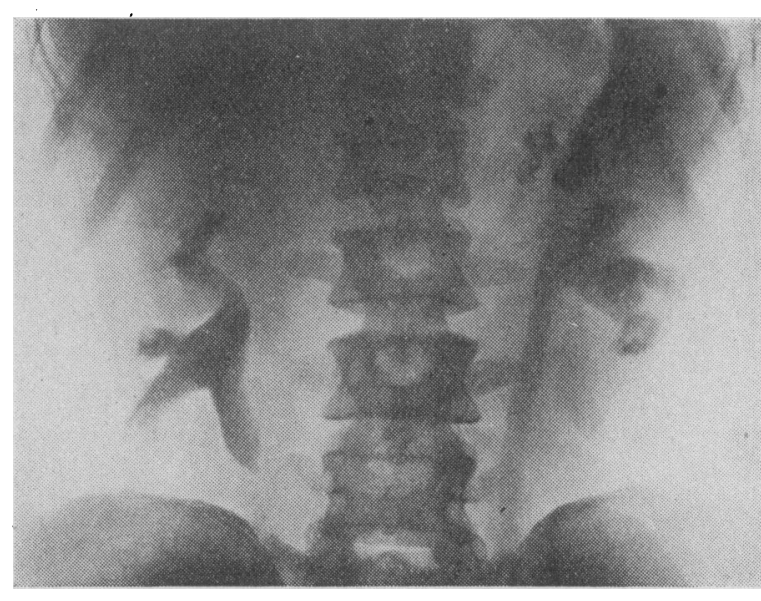

Case II (No. I) 


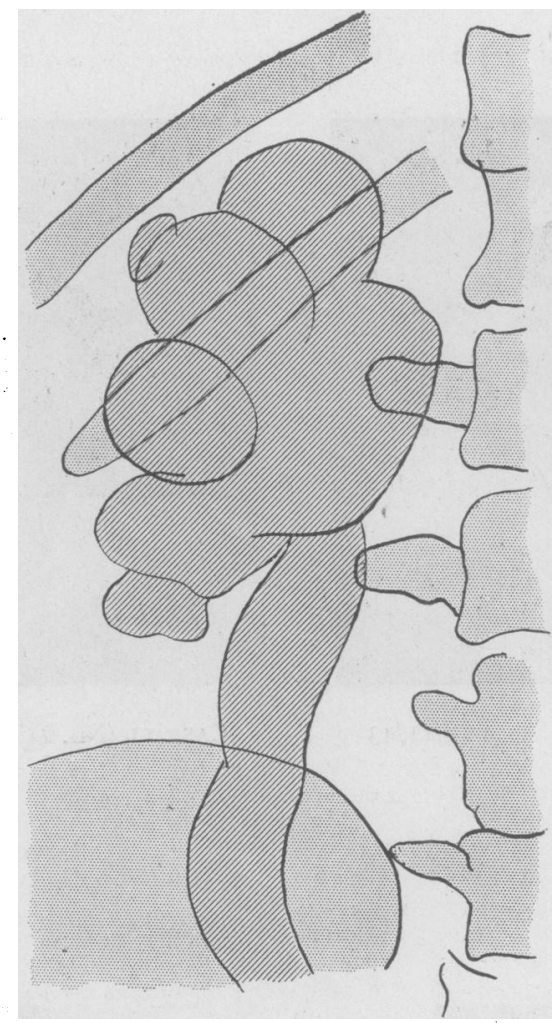

CAse I2,

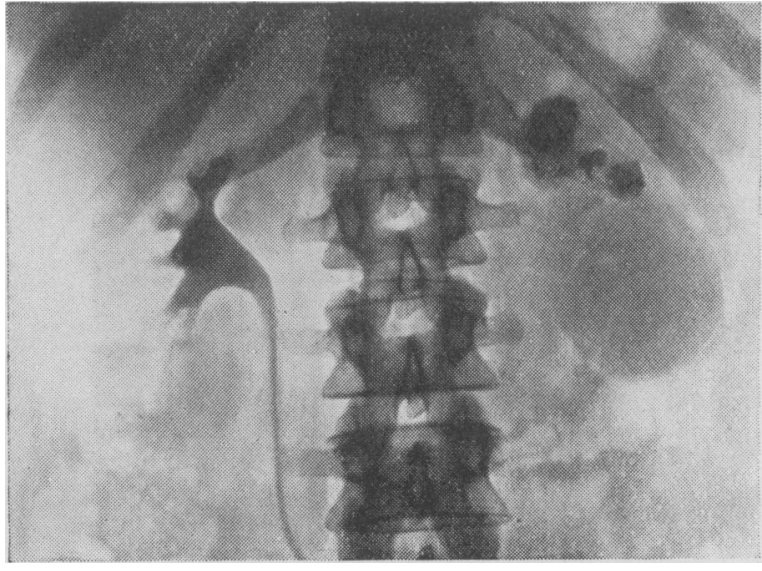

Case 14 
Discussion.

The rapid and complete healing of a deep ulcer in the bladder cannot always be counted upon, and this patient was undoubtedly fortunate.

CASE 2.

An R.A.M.C. officer was referred for an opinion upon a small mass in his epididymis. He complained of no urinary symptoms, and was apparently in robust health. X-ray with I.V. pyelography disclosed a partially disorganised right kidney. Guinea-pig inoculation tests from this urine came back positive.

Discussion.

This example is typical of a story which can be repeated time and again.

\section{CASE 3 .}

This patient had an acute attack of epididymitis. Pus cells and a small number of coliform organisms were found in his specimen, and he responded well to sulphathiazole. After a few weeks a similar attack occurred in the other side, and by this time the resolving epididymitis was suggestive of tuberculosis. Tubercle bacilli were not apparent in the specimen, but whilst a guinea-pig test was in progress X-ray revealed a destructive lesion in the right kidney.

Discussion.

Compare case 4 , which is very similar except that in this instance the acute phase settled down.

CASE 4.

This patient attended hospital suffering from acute epididymitis. Conservative measures having failed, the scrotum was incised. The wound continued to discharge until the necessity for orchidectomy became obvious. Only when the organ was sectioned and microscoped did its tuberculous nature become apparent. In the meantime the patient had been discharged from hospital. He was recalled, $\mathrm{X}$-rayed, and found to have a completely caseated and calcified left kidney, which was removed.

Discussion.

This case illustrates -

I. The possibly acute nature of a tuberculous lesion.

2. The necessity (overlooked at the time!) of considering the likelihood of a specific infection in any case which fails to respond in the usual way to the usual treatment.

3. The unusual phenomenon of extension of tuberculous epididymitis to the body of the testicle.

4. The necessity for seeking a renal focus-possibly latent-in every case of tuberculous epididymitis.

5. The risk of extension of active disease from an apparently quiescent focus, for this was a typical example of "autonephrectomy."

\section{CASE 5 .}

At the age of fourteen this child began to complain of pain in the region of the left kidney. I saw her for the first time a year later, when there was albuminuria. It was said that tubercle bacilli had been found in the urine. Uroselectan was normal, and cystoscopy negative. Retrograde pyelograms were also normal The patient was discharged with instructions to return for review in six months. The chest was normal. After six months these investigations were repeated and a positive specimen was obtained from the bladder and from the left ureter. The pyelograms remained anatomically normal, and there was no visible change in the bladder. These investigations have been repeated at regular intervals for nearly four years, during which time the girl has grown up and remains in tolerably good health. There has been no change at all in the condition in the urinary tract. A rather doubtful pulmonary lesion has been detected at the right apex.

\section{Discussion.}

This is apparently an example of tuberculous "bacilluria" associated with a pulmonary lesion. Unusual features are the appearance of tubercle bacilli in the urine at a time when the lesion in the chest was unsuspected and the persistence of the renal leakage over so long a period. In the absence of any change whatever in the pyelogram or the bladder, there is no indication for nephrectomy.

CASE 6.

First seen January I94I with urethral stricture. This was successfully dilated with some improvement. Continued symptoms were attributed to cystitis. In March I94I pus and b. coli were found; in April pus remained, but the coliform organisms were gone. In May $194 \mathrm{I}$ uroselectan was given and cystoscopy done again. The left orifice was pin-hole; the right orifice lay in an inflamed area, but did not show intrinsic disease. Neither could be catheterised. Tubercles were seen in the bladder, and tubercle bacilli subsequently found in the specimen. Repeated examinations have been made, and I.V.P.s in January 1942 and September 1943 are illustrated. There is (I) dilatation of both ureters in their lower parts, (2) hydronephrosis, of the right renal pelvis with probable scarring of the lowest major calyx, (3) calcification in the upper pole of the left kidney and changes which suggest an ulcerative lesion communicating with the upper calyx.

\section{Discussion}

Stricture and a mixed infection obscured the original diagnosis. Subsequent investigations suggest bilateral disease, but the condition seems stationary (?), and the general condition remains excellent. The final diagnosis has not been made. 
CASE 7.

Straight through X-ray shows a shadow in the region of the upper pole of the left kidney. Intravenous pyelography confirms that this is thrown by a stone. The deformity of the calyx is very typical-indeed is a caricature-of a tuberculous lesion beginning in the upper pole.

Clinically there was increased frequency (every five minutes) of micturition, but tubercle bacilli had not been found after a careful search.

\section{Discussion.}

A wrong diagnosis of increased frequency in some way related to renal calculus might have been made. The intravenous pyelogram was, however, suggestive of tuberculosis. Cystoscopic examination followed and a tuberculous ulcer was found on the trigone. The right orifice was healthy; the left was not clearly seen. Dye excretion was equal and good. Both ureters were catheterised; specimens segregated and retrograde pyelograms made. These examinations confirmed the diagnosis of left renal tuberculosis. Neither the intravenous nor the retrograde pyelogram of the right side is quite normal, but taken together they do not suggest any destructive lesion.

When the left kidney was exposed at operation it was externally normal, but was removed, found tuberculous with a small free stone.

Six months later the frequency was relieved from five minutes down to six times at night and the I.V.P. on the right unchanged. Twelve months after operation the specimen was clear and the frequency down to three at night.

\section{CASE, 8 .}

Straight X-ray shows a small group of stones in the region of the cortex. Intravenous pyelogram demonstrates a healthy kidney with prolongation of the middle calyx to reach the group of stones. Tubercle bacilli had not been diligently sought for in the specimen, which had contained a very occasional leucocyte. A provisional diagnosis of diverticulum of the calyx (9) was made and operation was undertaken for the relief of pain. The pocket of stones was located and excised with diathermy, the deficiency being closed by means of sutures. Subsequent microscopic examination exposed the tuberculous nature of the lesion and the kidney was removed. Further microscopic studies show that the disease was extending from the cortex into the medulla, and in addition two or three new areas of caseation had appeared under the capsule. Sections taken at random showed the remainder of the renal substance to be perfectly healthy.

\section{Discussion.}

This case illustrates the ease with which one may be led into a wrong diagnosis by an unusual feature 'such as the early formation of a stone or group of stones.

\section{CASE 9.}

This patient was in a sanatorium on account of tuberculous disease of the hip. Calculi were found in the left kidney, and an operation undertaken for their removal. A nephrostomy was done on account of sepsis. Progress was unsatisfactory, and eventually the kidney was removed. After removal microscopic sections showed that the kidney was tuberculous.

\section{Discussion.}

This was an early case in which insufficient attention was paid to detail. The presence of calculi and secondary infection were allowed to obscure the true diagnosis. It is none the less probable that the formation of these calculi was encouraged by decubitus.

\section{CASE ro.}

The pyelogram shows hydronephrotic dilatation in the lower part with a filling defect in the upper part. The ureter enters the pelvis at a high level.

\section{Discussion.}

The diagnosis was sufficiently obvious from the urinary findings, etc., but the pyelographic appearances were regarded as unusual, and an explanation was looked for when the kidney was examined. We found that the lower part was, indeed, hydronephrotic and not diseased. The irregularity at the upper pole was caused by fibrosis resulting in a filling defect, and in the hitching up of the ureter to an unusually high level with incidental obstruction and consequent hydronephrosis of the healthy part of the organ. The specimen is illustrated. (Opposite p. 267.)

\section{CASE II.}

Pre-operative $\mathrm{X}$-rays show calcification in the left kidney, which is completely disorganised. The right kidney is hydronephrotic and the ureter is grossly dilated. In one picture, thickening of the bladder wall, where it constricts the lower end of the right ureter, is clearly visible. There were tubercle bacilli in the urine and epididymitis. No attempt at cystoscopy was made.

\section{Discussion.}

This man had been twice refused operation at different hospitals on the ground of bilateral disease. In fact, the changes on the right side are due to back pressure only. The absence of a destructive lesion in the right pyelogram is the significant point. 
Left nephro-ureterectomy was performed. The left testicle was also diseased and was delivered through the lower end of the abdominal incision and its epididymis taken away. Three months later he was back at work. Twelve months after operation he still had nocturnal frequency, and tubercle bacilli were present. The I.V.P. on the right was unchanged.

\section{CASE 12.}

The intravenous pyelograms provide the sole evidence in this case. The left kidney is completely disorganised and functionless. The right throws a very faint shadow and is grossly hydronephrotic. It is, however, not calcified, and there is no frank ulcerative lesion seen. A tracing only can be reproduced as the shadow was so poor.

\section{Discussion.}

A diagnosis of ulilateral disease was made and nephrostomy performed on the right for the relief of oliguria with uraemia (N.P.N. $200 \mathrm{mgms}$. per cent). The specimen drained was found free from tuberculous infection. Subsequently the left kidney was removed. No decision has yet been reached about the final disposition of the right ureter, which will most probably be implanted into the colon. The N.P.N. has come down to 50 mgms per cent in a month.

\section{CASE I3.}

Investigation in 1936 with positive findings of tuberculosis in the left kidney. Cathetcr specimens from the right ureter also contained tubercle bacilli. Retrograde pyelograms on this side were completely normal and removal of the left kidney was advised. Subsequent X-rays of the right kidney show that it remains strictly normal. There was also a tuberculous pyosalpinx for which a radical operation for pelvic infection was done. In January 1938 frequency was down to three times at night and a full bladder caused anal. and rectal spasm. No pus or organisms were found. An irritable area in the middle line just above the trigone could be seen through the cystoscope. Zinc ionisation failed to give any relief (contrast with case $\mathrm{s} 8$ ).

\section{Discussion.}

The post-operative history in this case has fully justified the original decision to disregard, in view of the normal pyelogram, the finding of tubercle bacilli in the specimen segregated from the right side.

\section{CASE I4.}

This patient attended complaining of pain in the back, and was found to have a large perinephric abscess. Direct X-ray showed calcification in the upper pole of the kidney. A diagnosis of tuberculous kidney with perinephric abscess was made and the abscess was drained. Cystoscopic examination failed to disclose any change whatever in the bladder, although a purulent urine could be seen escaping from the left orifice. A retrograde pyelogram was made on the right because the intravenous pyelogram had not been very satisfactory, and a normal structure was disclosed on this side. Some doubt was felt about the tuberculous nature of the condition because of the complete absence of changes in the bladder. Nephrectomy was completed with some difficulty, and a typically tuberculous kidney was found. The changes in the ureter like those in the bladder, were minimal.

\section{Discussion.}

This is one of the only two cases in the series in which advanced changes in the kidney were associated with a perfectly normal bladder. This affords a further illustration of the over-riding value of the $X$-ray evidence.

\section{CASE I5.}

This patient began to have pain in the left groin with pyrexia shortly after childbirth. She was admitted with $\mathrm{X}$-ray films showing hydronephrosis and a urine which contained much pus with $\mathrm{b}$. coli. Intravenous pyelograms in hospital showed intermittent spasm in the left pyelogram, with dilatation at the lower end of the left ureter, but no gross anatomical lesion. Cystoscopy revealed a small number of tubercles around the left ureteric orifice. Specimens were segregated for animal inoculation, and retrograde pyelograms were made but were inconclusive. Two months from the original examination a further retrograde pyelogram show destructive changes in the left kidney. By this time the guinea pig test was known to be positive and the left kidney and ureter were removed.

\section{Discussion.}

This case illustrates the converse of the argument elaborated in case 13. The cystoscopic appearances afforded the first positive evidence and started us off on the right track. Although the pyelograms are of the first importance one cannot afford to neglect the other methods of investigation.

\section{CASE 16.}

The X-ray shows calcification in the right kidney. Nephrectomy was undertaken and the kidney, which was abnormally low, was found to be densely adherent. The ureter was grossly dilated and full of necrotic material, but it also was so fixed as to be almost immovable. The patient was frail and not very young, and ureterectomy was out of the question. A moderately large tube was passed well down the ureter, sutured in position, and brought out at the lower angle of the wound. This was not finally removed for three or four weeks, by which time the track was well formed. After the tube had been removed, healing of the sinus occupied a further few weeks, but was never troublesome, and was finally sound and satisfactory. 


\section{Discussion.}

In a case of this type the whole kidney wound is likely to break down and remain unhealed if the dilated and infected ureter is allowed to discharge freely into it.

\section{CASE I7.}

An old history of tuberculosis of the spine (two lesions), peritoneum and epididymes. Investigation revealed a caseous left kidney which was removed in 1938. A year's sanatorium treatment followed because of the appearance of the second focus in the spine-this healed. An attack of right renal colic with oliguria in 1939 was probably due to the passage of a small stone. Bladder stones were crushed in I940, and a limited resection of the bladder neck was done in 1942. Very troublesome frequency persists, and there are still tubercle bacilli in the specimen, but the right kidney remains anatomically normal.

\section{Discussion.}

This case illustrates the confusion which may arise from intercurrent stone formation, whether or not due to decubitus. If these attacks are not recognised correctly, an unduly pessimistic attitude may be taken. Similarly, the continued appearance of tubercle bacilli does not indicate active disease in the remaining kidneys. This patient now still does in general practice a very much harder day's work than most people, and plays hard as well!

CASE I8.

Two years after nephrectomy this child had intolerable frequency of micturition. The specimen contained numerous red cells, a few leucocytes and no organisms. Intravenous pyelography displayed a normal left kidney and ureter with a small rounded bladder, and some residue after micturition. Cystoscopy displayed a generalised cystitis with superficial ulceration, but nothing characteristic of active tuberculosis. Ionisation with 2 per cent zinc sulphate in the bladder at fifteen milliamps for ten minutes was carried out. Two years later the child reported entirely free from all symptoms with a perfectly clear specimen without a trace of albumen.

\section{Discussion.}

This was an exceptionally successful case in which the response to ionisation was rapid and complete. I have had relief in a number of other cases, but never anything so dramatic as this.

\section{CASE I9.}

Right nephrectomy 1938 (elsewhere)-ureter divided "low down as possible"-cauterised and dropped back. The nephrectomy wound has never completely healed. August 1941, complains of constant dribbling micturition. N.P.N. 47 mgms. per cent--I.V.P. shows' hydronephrosis and hydro-ureter on the left with a small spastic bladder which empties imperfectly.

\section{Discussion.}

So great was the distress that transplantation to the colon was accepted. After a preliminary transverse colostomy (a clumsy method of isolating the lower bowel which I have since abandoned), the left ureter was successfully implanted into the colon by Bernard Ward's technique. Urine is voided from the bowel about every four hours.

In conclusion, I must thank Pr. J. H. Mather and the other radiologists responsible for the $\mathrm{X}$-raying of these patients, also the Medical Superintendents of the Lancashire and Cheshire County Hospitals at Wrightington and Clatterbridge respectively, and numerous other workers, in particular Dr. Dobson and Dr. Doreen Watt for their most valuable assistance in collecting the material.

\section{REFERENCES}

(1) WELLS, C. A., "Tuberculous Epididymitis," British Journal of Urology. 1938, 10, II4.

(2) MEDLAR, EDGAR M. "Cases of Renal Infection in Pulmonary Tuberculosis. Evidence of Healed Tuberculous Lesions," Amer. Jour. of Path., 1926, 2, 401.

MEDLAR, E. M., and SASANO, K.T. Amer. Review of Tuberculosis, 1924-25, 10, 370 .

(3) BAND, DÁ ID, ALSTON, J. M., GRIFFITHS, A. STANLEY, and MUNRO, W. T. "Renal Tuberculosis," Edin. Med. Jour., I935, 52, New Series IVth).

(4) COULAND, E. An address before the Societé Française d'urologie, April 1935. Reported in "Urology in r935." Paris Medical,

(5) BREM. Jour. Am. Med. Ass. 1909, 909.

(6) BEITZKE. Berl. Klin. Voch. I909, 909.

(7) MUNRO, D., Jour. of Urology, 1936, 36, 710.

(8) WELLS, CHARLES. "Tidal Drainage of the Bladder," British Journal of Urology. 1942, $14,73$.

(9) WELLS, C. A., "Diverticulum of Renal Pelvis," British Journal of Urology. I933, 5, 268.

It is regretted that the fourth article of this series, "The Relationship between Renal and Pulmonary Tuberculosis," has not appeared in time for publication.-Ed. 